\title{
ASTEROID POLARIMETRY: A PROGRESS REPORT
}

\author{
J. VEVERKA \\ Cornell University
}

This is a brief report on the present status of asteroid polarimetry. A detailed paper is in preparation jointly with $T$. Gehrels.

The first polarization measurements of asteroids were made by Lyot (1934), who photographically determined the polarization curves of Ceres and Vesta. These curves are reproduced by Dollfus ${ }^{1}$ (1961). Unfortunately, because of the low sensitivity of the photographic method, they do not agree very well with recent photoelectric measurements.

The first photoelectric polarization measurements of asteroids were made by Provin (1955) (details of this work are given by Dollfus, ${ }^{1} 1961$ ), and in recent years this work has been extended by Gehrels (unpublished) and by Veverka (1970). To date, fairly complete polarization curves for about a dozen asteroids have been obtained, and at least partial data are available for twice that number. Invariably, all asteroids for which sufficient data have been accumulated show lunarlike polarization curves with well-developed negative branches. This suggests that asteroid surfaces generally consist of a microscopically intricate, porous material in which multiple scattering is not dominant (Lyot, 1929). But, although these curves have similar shapes, they can differ considerably in detail (fig. 1). It is clear from figure 1 that, for example, the polarization curves of Ceres and Vesta are significantly different from each other, and from that of the Moon. It is therefore reasonable to suppose that some information about the composition and texture of asteroid surfaces can be obtained by matching these curves in the laboratory. Such work is now in progress using powdered meteorite surfaces.

Asteroid polarization curves are also phenomenologically useful because from them one can estimate the absolute reflectivities of the surfaces. For dark, texturally complex surfaces, $h$, the slope of the linear part of the polarization curve beyond the inversion angle, seems to be inversely correlated with surface reflectivity. This relationship has been exploited by KenKnight et al. (1967), Widorn (1967), Gehrels et al. (1970), and others. Veverka (1971a) gives a short discussion of this method. Presently, the drawback is that this relationship has been calibrated adequately only for lunar regions and for

\footnotetext{
${ }^{1}$ See p. 96.
} 


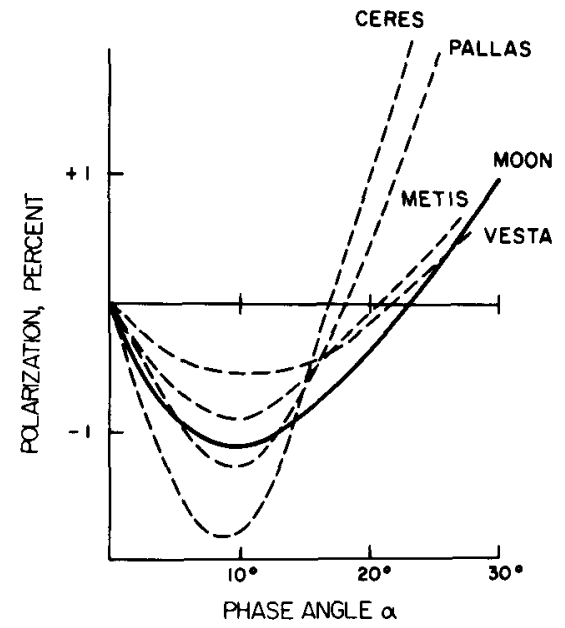

Figure 1.-A comparison of the polarization curves of the Moon and four asteroids. The lunar curve is from Lyot (1929). The asteroid curves are based on previous measurements as follows: Ceres and Pallas from Provin (1955; details given in Dollfus, 1961), Gehrels (unpublished), and Veverka (1970); Vesta from Gehrels (unpublished) and Veverka (1970); and Metis from Veverka (1970). The measurements by Gehrels were made either at 0.52 or $0.56 \mu \mathrm{m}$; all others were made in integrated light. Note that from Earth few asteroids can be observed at phase angles larger than $30^{\circ}$.

possible lunar materials. But in view of the results of McCord, Adams, and Johnson $^{2}(1970)$ it may be more appropriate to calibrate the relationship by using pulverized meteorite surfaces. Nevertheless, the calibration now available is probably adequate to yield reasonable estimates of asteroid reflectivities. For example, one finds in this way $0.25 \pm 0.07$ for the reflectivity of Vesta (Veverka, 1971a) and $0.16 \pm 0.03$ for that of Flora (Veverka, 1971b), both in visible light.

An interesting possibility being investigated in the laboratory is that for dark, microscopically intricate surfaces, the minimum of the negative branch of the polarization curve $P_{\min }$ may be a crude indicator of surface reflectivity. Data for volcanic cinders and ashes by Lyot (1929) suggest that this may be the case (the deeper the negative branch, the lower the reflectivity). If such a relationship could be established, even approximately, it would provide a means of estimating the absolute reflectivity of an asteroid from a single measurement at a phase angle near $10^{\circ}$. This would be of special importance in the case of Trojan asteroids because they cannot be observed at sufficiently large phase angles to determine $h$, and there is at present no reliable way of estimating their reflectivities. (See, for example, Dunlap and Gehrels, 1969.) Furthermore, Trojans are faint and difficult to locate, making the determination of a complete polarization-phase curve a formidable task.

${ }^{2}$ See p. 51 . 
It has been suggested (Gehrels and Teska, 1963) that simultaneous polarization and brightness measurements can be used to decide conclusively to what extent the short-period brightness fluctuations of asteroids are due to changes in surface reflectivity, rather than to changes in the projected surface area. The idea is to observe at large phase angles where the polarization should be positive and inversely correlated to surface reflectivity (Gehrels, 1970). Such observations are difficult because at large phase angles asteroids are not well placed in the sky, and can be observed for only a few hours, making it impossible, in general, to follow a complete rotation during any one night. So far, no polarization-brightness variations related to rotation have been established, but only two asteroids have been observed simultaneously in brightness and in polarization: Pallas (Gehrels, unpublished) and Eunomia (Veverka, 1970).

\section{ACKNOWLEDGMENTS}

I wish to thank T. Gehrels, W. Liller, C. Sagan, and F. L. Whipple for helpful discussions. Some of the observations reported in this paper were made at Harvard. The Harvard polarimeter project is supported by AFOAR contract no. F19-628-68-C-0228. This work was supported in part by NASA NGR 33-010-082.

\section{REFERENCES}

Dollfus, A. 1961, Polarization Studies of Planets. Planets and Satellites (eds., G. P. Kuiper and B. M. Middlehurst), ch. 9. Univ. of Chicago Press. Chicago.

Dunlap, J. L., and Gehrels, T. 1969, Minor Planets. III. Lightcurves of a Trojan Asteroid. Astron. J. 74, 796.

Gehrels, T. 1970, Photometry of Asteroids. Surfaces and Interiors of Planets and Satellites (ed., Dollfus), ch. 6. Academic Press, Inc. New York.

Gehrels, T., Roemer, E., Taylor, R. C., and Zellner, B. H. 1970, Minor Planets and Related Objects. IV. Asteroid (1566) Icarus. Astron. J. 75, 186.

Gehrels, T., and Teska, T. M. 1963, The Wavelength Dependence of Polarization. Appl. Opt. 2, 67.

KenKnight, C. E., Rosenberg, D. L., and Wehner, G. K. 1967, Parameters of the Optical Properties of the Lunar Surface Powder in Relation to Solar Wind Bombardment. J. Geophys. Res. 72, 3105.

Lyot, B. 1929, Studies of the Polarization of Planets. NASA TT F-187.

Lyot, B. 1934, Polarisation des Petites Planètes. Bull. Astron. 67, 3.

McCord, T. B., Adams, J. B., and Johnson, T. V. 1970, Asteroid Vesta: Spectral Reflectivity and Compositional Implications. Science 168, 1445.

Provin, S. 1955, Preliminary Observations of the Polarization of Asteroids (abstract). Publ. Astron. Soc. Pac. 67, 115.

Veverka, J. 1970, Photometric and Polarimetric Studies of Minor Planets and Satellites. Ph. D. Thesis. Harvard Univ.

Veverka, J. 1971a, The Polarization Curve and the Absolute Diameter of Vesta. Icarus 15, 11.

Veverka, J. 1971b, Photopolarimetric Observations of the Minor Planet Flora. Icarus 15(3), in press.

Widorn, T. 1967, A Photometric Method of Estimating the Diameters of Minor Planets. Ann. Univ. Sternwarte Wien 27, 112. 


\section{DISCUSSION}

GEHRELS (added after the conference): I think it dangerous in principle to calibrate with meteoritic material; one is then likely to derive meteoritic characteristics. The calibration should, instead, be done with asteroids and perhaps satellites. We must obtain good diameter measurements, possibly from space missions. 